\title{
TTR
}

Traduction, terminologie, re?daction

\section{Hans J. Vermeer (1996). Das Übersetzen im Mittelalter (13. und 14. Jahrhundert). Heidelberg, TEXTconTEXT (Band 4.1-4.3)}

\section{Nadja Grbić et Michaela Wolf}

Volume 12, numéro 2, 2e semestre 1999

Poésie, cognition, traduction II - Autour d'un poème de W. H. Auden

Poetry, Cognition, Translation II — On a Poem by W. H. Auden

URI : https://id.erudit.org/iderudit/037384ar

DOI : https://doi.org/10.7202/037384ar

Aller au sommaire du numéro

Éditeur(s)

Association canadienne de traductologie

ISSN

0835-8443 (imprimé)

1708-2188 (numérique)

Découvrir la revue

Citer ce compte rendu

Grbić, N. \& Wolf, M. (1999). Compte rendu de [Hans J. Vermeer (1996). Das Übersetzen im Mittelalter (13. und 14. Jahrhundert). Heidelberg, TEXTconTEXT (Band 4.1-4.3)]. TTR, 12(2), 200-201. https://doi.org/10.7202/037384ar d'utilisation que vous pouvez consulter en ligne.

https://apropos.erudit.org/fr/usagers/politique-dutilisation/ 
Hans J. Vermeer (1996). Das Übersetzen im Mittelalter (13. und 14. Jahrhundert). Heidelberg, TEXTconTEXT (Band 4.1-4.3)

La quatrième publication de la collection représente la suite des "Esquisses d'une histoire de la traduction" ("Skizzen zu einer Geschichte der Translation ») de Vermeer, publiées en 1992. Dans cet ouvrage en trois volumes, Vermeer retrace l'histoire de la pratique de la traduction en vigueur dans l'Europe occidentale du XIII et du XIV siècles et fait un survol intéressant, bien que partiel, d'une époque où la réflexion traductologique n'était guère présente et qui mériterait de faire l'objet de recherches particulières. Estimant que le choix des 
sujets relève de la disponibilité de la documentation, Vermeer regrette particulièrement le manque de données sur l'Europe de l'Est.

Dans le volume 1, Vermeer décrit la situation de la recherche et, à partir des deux volumes de 1992 qui offrent comme les fils rouges de cette entreprise, il présente une rétrospective des stratégies dominantes allant de la période mésopotamienne jusqu'à la fin du XII siècle. Bien que les livres se réfêrent à l'époque du Haut Moyen-Âge, Vermeer élargit le champ de recherche jusqu'au XIX ${ }^{\mathfrak{e}}$ siècle. En effet, malgré certaines différences, Vermeer considère cette période comme une époque assez homogène, puisque le changement de paradigme vers une histoire des idées n'aura lieu qu'au XIX ${ }^{\mathfrak{e}}$ siècle, changement produit par la dynamisation de la théorie, en particulier de la théorie humboldtienne de la vision du monde.

À partir de paramètres comme le type de texte (scientifique, littéraire, biblique), le public cible (experts, amateurs) et la fonction (compréhension, édification chrétienne, divertissement, imitation), Vermeer établit une liste des stratégies de traduction pour revenir ensuite à ses Esquisses qu'il divise en parties principales : linguistique ou philosophie du langage, rhétorique, littérature spécialisée, didactique des langues étrangères, opinion des traducteurs.

Après avoir présenté les aspects de la philosophie du langage, de la linguistique et de la rhétorique, il se consacre au sujet proprement dit de son premier volume, à savoir le Moyen-Âge arabe et latin et la traduction de l'épopée séculaire, la Bible et les textes scientifiques. Il décrit les centres de traduction comme Tolède, Salerne et autres, les écoles de traduction et les traducteurs particuliers. Enfin, Vermeer aborde le statut de l'interprétariat au Moyen-Âge et de la traduction en langues vernaculaires.

Le second volume s'intitule l'Allemand comme langue cible et examine dans quelle mesure les traductions ont contribué au développement littéraire dans le Haut Moyen-Âge, soit par l'introduction de nouveaux sujets (par exemple, l'épopée du roi Arthur), par le renforcement de tendances vernaculaires (par exemple, la légende), par l'apparition de nouveaux courants d'idées, etc. À la fin du volume, le lecteur trouvera une sélection de citations puisées dans des ceuvres germanophones du Moyen-Âge traitant de traduction. Une bibliographie extrêmement détaillée est fournie par Helga Ahrens, ainsi qu'un index des trois volumes. 
Wolfram Wilss. Translation and Interpreting in the 20th century : Focus on German. Amsterdam/Philadelphia, John Benjamins, 1999, $258 \mathrm{p}$.

Une fois de plus, Wolfram Wilss comble une lacune en traductologie: l'histoire de la traduction et de l'interprétation en Allemagne de 1900 à nos jours.

Dans sa préface, l'auteur précise bien qu'il s'agit de donner aux lecteurs un aperçu du développement de la médiation langagière $\mathrm{du}$ $\mathrm{XX}^{\mathrm{e}}$ siècle. Et ceci en Allemagne. Le livre est structuré de telle façon que le lecteur pressé peut faire un choix de lectures sans être tenu de lire l'ouvrage du début à la fin. Le fait que l'auteur sait marier la théorie à la pratique ressort dès la lecture de la table des matières, car les deux volets reçoivent un traitement égal. Comme presque toujours, Wilss traite toute problématique de façon aussi neutre que possible : il justifie ses prises de position, qu'elles soient positives ou négatives. Wilss est en outre très soucieux de ses lecteurs : il leur fournit une bibliographie des plus récentes ainsi qu'un index par nom d'auteurs et par sujets.

Le chapitre 1, Rétrospective, contient, entre autres, un certain nombre d'anecdotes de l'histoire de la traduction avant l'ère qui nous interesse ici. Le lecteur voit ainsi défiler devant ses yeux les noms de ceux qui ont fait l'histoire de la traduction, cette histoire qui s'écrit avec un grand " $\mathrm{H}$ ", tels que Cicéron, saint Jérôme et Luther. Ce même chapitre fournit également l'explication de certaines notions fondamentales. C'est ainsi que l'acte de traduire est subdivisé en trois catégories : a) la traduction de textes bibliques; b) la traduction de textes littéraires; c) la traduction de textes spécialisés. Subdivision tripartite également pour l'interprétation.

Une fois cela établi, les chapitres 2,3 et 4 traitent de périodes bien définies : 1900-1919; 1919-1945; 1945-1990, respectivement. Le sous-chapitre 2.1 qui est intitulé The Auswärtige Amt in Bonn (L'Office des affaires étrangères), nous apprend que jusqu'en 1918, le mot dragoman servait à désigner les interprètes pour les langues exotiques. La formation de ces dragomans nous est décrite en détail et 